\title{
Estrategia didáctica para enseñar a planificar los procesos de enseñanza y aprendizaje de la matemática
}

Pérez González, Andel; Valdés Rojas, Martha Beatriz; Garriga González, Ana Teresa

Estrategia didáctica para enseñar a planificar los procesos de enseñanza y aprendizaje de la matemática Revista Educación, vol. 43, núm. 2, 2019

Universidad de Costa Rica, Costa Rica

Disponible en: http://www.redalyc.org/articulo.oa?id=44058158016

DOI: https://doi.org/10.15517/revedu.v43i2.32236

Esta obra está bajo una Licencia Creative Commons Atribución-NoComercial-SinDerivar 3.0 Internacional. 


\section{Estrategia didáctica para enseñar a planificar los procesos de enseñanza y aprendizaje de la matemática}

\section{Didactic Planning Strategies for Teachers of Mathematics}

Andel Pérez González

Universidad "José Marti Pérez", Sancti Spiritus, Cuba andelperezgonzalez16@gmail.com

(iD http://orcid.org/0000-0003-4435-4030

Martha Beatriz Valdés Rojas

Universidad "José Martí Pérez", Sancti Spiritus, Cuba

mrojas@uniss.edu.cu

(iD) http://orcid.org/0000-0002-7593-6140

Ana Teresa Garriga González

Universidad "José Martí Pérez", Sancti Spiritus, Cuba

atgarriga@uniss.edu.cu

(iD) http://orcid.org/0000-0002-7722-4606
DOI: https://doi.org/10.15517/revedu.v43i2.32236

Redalyc: http://www.redalyc.org/articulo.oa?id=44058158016

Recepción: 05 Febrero 2018

Aprobación: 10 Mayo 2019

\section{Resumen:}

En este artículo se presenta una propuesta de estrategia didáctica para enseñar a planificar los procesos de enseñanza y aprendizaje de la Matemática a un futuro cuerpo docente de esta asignatura considerando el enfoque integrador de las habilidades: analizar metodológicamente unidades didácticas, diseñar sistemas de clases y planificar clases. Este resulta de una investigación cuantitativa en la que se fundamenta teóricamente la habilidad profesional planificar los procesos de enseñanza y aprendizaje de la Matemática, se presenta una estrategia didáctica diseñada con el objetivo de perfeccionar el proceso de formación y desarrollo de la mencionada habilidad profesional y se analizan los resultados de la aplicación de esta en la práctica.

Palabras ClaVE: estrategia didáctica, habilidades profesionales, planificación.

\section{Abstract:}

This article proposes a didactic planning strategy aimed at student teachers of Mathematics and considers an integrative skills approach which includes methodological analysis, didactic units, class design and planning systems. The data obtained is based on the results of a quantitative study that has, as its theoretical basis, general professional skills involved in planning Math Classes. Teaching strategies are also suggested designed to perfect learning and development of the aforementioned professional skills and the results analyzed after they are put into practice.

KEYWORDS: Didactic Strategy, Professional Skills, Planning.

\section{UNA INTRODUCCIÓN NECESARIA A LA PROBLEMÁTICA}

En los últimos años las sistemáticas transformaciones que distinguen la economía, la cultura y las relaciones políticas y sociales a nivel internacional, nacional y local exigen nuevos roles a las universidades. Desde esta realidad, se reconoce por Saravia y Flores (2005) que el desafío de la formación inicial de los educadores está: "en la estructuración de un sistema que sea creativo, flexible y crítico, abierto a los constantes cambios, a los aportes de la ciencia, la cultura y centrados en la realidad y su contexto” (p. 36); es decir capaz de formar y desarrollar en el estudiantado sus habilidades profesionales para cumplir con éxito sus funciones. 
Como respuesta a esta realidad Massón, Llivina y Arencibia (2011) alegan que "existe una concepción que sostiene que el docente principalmente debe estar formado para el cómo enseñar" (p. 80). Esta idea destaca la importancia de la preparación del alumnado de las carreras pedagógicas para la planificación de los procesos de enseñanza y aprendizaje; temática que se enriquece sistemáticamente a nivel internacional y nacional según las particularidades de cada asignatura.

Insatisfechos con los resultados que se logran en la práctica el objetivo del artículo es: proponer una estrategia didáctica para el perfeccionamiento de la formación y el desarrollo de la habilidad profesional planificar los procesos de enseñanza y aprendizaje de la Matemática.

Este se organizó en tres apartados que incluyen los fundamentos teóricos relacionados con la formación y desarrollo de las habilidades profesionales, la caracterización de la habilidad profesional objeto de análisis y la estrategia didáctica, acompañada de los resultados de su aplicación práctica.

La metodología utilizada se caracterizó por un enfoque cuantitativo, el cual exigió la utilización de métodos teóricos como el histórico-lógico, el analítico-sintético y el inductivo-deductivo; estos permitieron determinar y construir los posicionamientos teóricos a considerar durante el diseño de la estrategia didáctica. Igualmente se utilizaron métodos empíricos como el análisis del producto de la actividad y la observación pedagógica; ellos facilitaron la recogida y el análisis de los datos que ilustran los resultados de la aplicación de la estrategia en la práctica pedagógica.

\section{MARCO TEÓRICO PARA LA FORMACIÓN Y DESARROLLO DE LAS HABILIDADES PROFESIONALES}

La revisión bibliográfica permitió plantear en principio que la formación de habilidades es un tema de actualidad en la práctica educativa de estos tiempos; al respecto la Oficina Regional de Educación para América Latina y el Caribe (Unesco), destacó la importancia de desarrollar las que se relacionan con el desempeño profesional como una tendencia contemporánea la cual reconoce la resolución de los problemas que se presentan a lo largo de la vida profesional.

Sobre la categoría habilidad estudiosos clásicos como: Savin (1972), Lerner y Skatkin (1980), Petrovsky (1980) y Danilov (1985) resaltan el papel de las acciones y operaciones sobre la base de la experiencia de los sujetos, y las relaciones entre ellas; la utilización creadora de los conocimientos al ejecutar actividades teóricas y prácticas; así como el dominio de un sistema de acciones psíquicas y prácticas necesarias para regular la actividad con ayuda de los conocimientos que la persona posee.

Igualmente, autores cubanos como Brito (1984) y López (1990) destacan la relación de las habilidades con la actividad y el papel de las acciones y operaciones como necesarias para su regulación. Estas posiciones se enriquecen al considerar que las habilidades no son más que las acciones desarrolladas sobre la base de los conocimientos adquiridos al ponerlos en acción; según González, Castellanos, Córdova, Rebollar, Martínez, Fernández, Martínez y Pérez (1995).

Por otra parte, se precisa por Álvarez (2001) que las habilidades constituyen: “Un sistema de acciones y operaciones, dominado por el sujeto, que responde a un objetivo. Es el componente del contenido, que refleja las realizaciones del hombre en una rama del saber propio de la cultura de la humanidad" (p.16). El propio autor señala que para cada habilidad es posible determinar acciones y operaciones cuya integración permite el desarrollo de modos de actuación, criterio que revela la importancia de formar y desarrollar las habilidades desde posiciones integradoras e insiste en el papel de las acciones y operaciones al aplicar el conocimiento en una tarea específica.

En los criterios analizados se revela como regularidad la asimilación de las habilidades durante la actividad, su logro a partir de sistematizar acciones y operaciones, la influencia del contexto en dicho proceso y la necesidad de precisar el cómo dirigir su asimilación. En tal sentido, se comparten las posiciones de Álvarez (2001), pues comprende las habilidades desde la teoría de la actividad y destaca el papel de las acciones y operaciones a partir de la integración de los contenidos. 
Al profundizar en la formación y desarrollo de una habilidad se identifica por Talízina (1988) la necesidad de ofrecer la imagen generalizada de cada acción a ejecutar, criterio que se retoma al diseñar la estrategia didáctica que se propone. Igualmente, se considera que la formación de las habilidades más generales ocurre mediante la articulación sistémica de otras de orden menor, cuya sistematización posibilita su desarrollo, según Álvarez (1988).

Para López (1990) la formación de una habilidad se analiza, “(...) como la adquisición consciente de los modos de actuar, cuando bajo la dirección del maestro o profesor el alumno recibe la orientación adecuada sobre la forma de proceder" (p.2). Igualmente, López (1990) indica que el desarrollo:

Ocurre una vez adquiridos los modos de acción, se inicia el proceso de ejercitación, es decir, de uso de la habilidad recién formada en la cantidad necesaria y con una frecuencia adecuada, de modo que vaya haciéndose cada vez más fácil de reproducir o usar, y se eliminen los errores. (p. 2)

$\mathrm{Al}$ respecto, Bermúdez y Rodríguez (1996) plantearon que la formación de una habilidad condiciona su desarrollo y por eso es imposible separar totalmente las etapas analizadas y el valor metodológico de las estrategias a utilizar en cada caso, criterio que se tiene en cuenta en lo adelante. Es imprescindible entonces, que el estudiantado reconozca conscientemente las acciones que componen la habilidad y comprenda las operaciones de cada una de ellas.

Igualmente insisten en que formar y desarrollar una habilidad es un proceso complejo y dinámico el cual tiene requisitos cuantitativos y cualitativos. Los primeros determinan la frecuencia de ejecución y la periodicidad con que se dan sus acciones y operaciones. Los segundos precisan la complejidad y flexibilidad de la ejecución de las acciones y operaciones según el contexto de actuación.

Desde la perspectiva de este escrito se enfatiza en la complejidad e interrelación de ambos procesos, pues la formación de una habilidad necesita de su desarrollo para lograr su continuidad, a la vez que para el desarrollo se requiere de un sistemático proceso de formación que perpetúe su continuidad en diferentes contextos. En tal sentido, para desarrollar una habilidad Barreras (2003) considera necesario:

- Trabajar las ejecuciones simples y las complejas en orden, según el grado de dificultad de los conocimientos, el contexto y su complejidad.

- Distribuir temporalmente las acciones y las operaciones para no ejecutarlas ni muy separadas ni muy cercanas; o sea graduar su periodicidad.

- Una frecuencia adecuada del número de veces que se presenta cada acción.

- La variabilidad de los conocimientos y los contextos de actuación en que son aplicadas las habilidades desde posiciones flexibles.

- Retroalimentar el resultado de la sistematización de la habilidad para su perfeccionamiento a partir de valorar los errores y corregir las acciones.

- Evitar el cansancio, la monotonía y la fatiga que disminuyen la capacidad de trabajo y conspiran contra la adquisición de las habilidades.

- Fomentar la motivación y la conciencia, como factores imprescindibles que facilitan la adquisición de las ejecuciones.

Desde la Didáctica General Ginoris, Addine y Turcaz (2006) reconocen como requerimientos para la formación y desarrollo de una habilidad:

(...) el conocer el objetivo y la esfera de aplicación de las habilidades; comprender las particularidades de los objetos y fenómenos que constituyen fuentes de los conocimientos con los cuales se interactúa; conocer el contenido y la secuencia de acciones u operaciones; demostrar la aplicación de la secuencia de acciones u operaciones; aplicar de manera conjunta con el profesor y otros alumnos esta secuencia de acciones u operaciones; realizar un proceso de ejercitación para perfeccionar la ejecución, y eliminar errores y acciones u operaciones realmente innecesarias y aplicar con independencia la secuencia de acciones u operaciones en nuevas situaciones docentes (p.33). 
Además de los señalados, se apunta que es preciso considerar el papel de la esfera afectivo-motivacional para hacer más consciente los momentos descritos. Es por ello que debe ser un requisito la comprensión y aplicación de las relaciones entre las acciones y operaciones que conforman una habilidad.

Desde estos puntos de vista se precisa que la formación y desarrollo de una habilidad ocurre en la actividad y que el estudiantado debe conocer cómo proceder a partir de sus carencias y potencialidades, y las acciones y operaciones a efectuar. $\mathrm{Al}$ respecto, Ruiz (2014) planteó que para estructurar una habilidad en acciones es necesario hacerlo con las instrumentaciones ejecutoras esenciales, ya que una vez sistematizadas constituyen las invariantes funcionales, es decir, por las cuales tiene que transcurrir la ejecución de la actuación, criterio que comparten los autores del artículo.

Se confirma así la complejidad de la formación y el desarrollo de las habilidades en la actividad educativa, desde su doble carácter, es decir, como actividad práctica e intelectual en la cual, de manera protagónica, interviene el estudiante bajo la atención del cuerpo docente. Este proceso es individual y consciente, lo cual determina la importancia de la actuación del profesorado tanto en su formación como en su desarrollo.

Desde la perspectiva de González, Vega y Francisco (2015) el proceso de formación y desarrollo de una habilidad se planifica, organiza, ejecuta y controla. La planificación consiste en determinar las habilidades terminales y sus invariantes. La organización establece cuándo y con qué conocimientos se ejecutan las acciones y operaciones. Durante la ejecución se orientan las acciones y operaciones que corresponden y se proponen como objetivo a lograr; el control establece una escala analítico-sintética para la evaluación de los indicadores de las diferentes operaciones de la habilidad.

$\mathrm{Al}$ profundizar en la clasificación de habilidades se comprobó que, desde el punto de vista más general, según el modo de obtener y aplicar el conocimiento por un trabajador, Miari (1982) consideró las intelectuales, sensoriales y prácticas; según la ejecución de la actividad humana y su nivel de incidencia en la elaboración de diversas actividades, González et ál. (1995) las diferenció en generales y específicas, y Barreras (1997) como intelectuales, prácticas, generales y particulares.

En todos los casos se refieren a las profesionales, pero no concretamente para el campo de la educación. Para este escenario, en particular, se reconocen según las acciones por desarrollar: las habilidades específicas, las lógicas y las del procesamiento de la información y la comunicación. Las primeras según Fuentes y Álvarez (1998) son:

El tipo de habilidad que el sujeto desarrolla en su interacción con un objeto de estudio o trabajo concreto y que en el proceso de enseñanza-aprendizaje, una vez que son suficientemente sistematizadas y generalizadas, se concretan en métodos propios de los diferentes objetos de la cultura que se configuran como contenido (p.107).

Los estudiosos de las habilidades profesionales plantean que su formación y desarrollo aseguran la ejecución de las actividades propias de la profesión al tener que efectuar las acciones y operaciones necesarias para solucionar los problemas profesionales de forma consciente. En este sentido, se considera que toda profesión se desglosa en un sistema de habilidades definidas en tareas concretas que deben saber y saber hacer los sujetos, desde su formación inicial.

Las habilidades profesionales son analizadas por Miari (1982) como la: "disposición a efectuar la acción o el conjunto de acciones productivas de manera consciente, utilizando correctamente, en situaciones dadas, los métodos oportunos de su realización, logrando adecuados resultados cualitativos y cuantitativos en el trabajo" (p. 66), criterio donde se otorga un papel relevante al sujeto y a los métodos para desarrollar las acciones, así como a su planificación anticipada.

Para Márquez (1987) las habilidades profesionales de la persona educadora:

Manifiestan el dominio satisfactorio de acciones prácticas e intelectuales que garantizan el éxito en la ejecución de actividades de la profesión pedagógica, que se adquieren fundamentalmente sobre la base de los conocimientos asimilados en la preparación pregraduada y que se perfeccionan con el ejercicio de la profesión y la superación postgraduada (p. 11). 
Este criterio destaca su importancia para cumplir las funciones y resolver situaciones de la profesión, al tener como base los problemas profesionales.

Este criterio destaca su importancia para cumplir las funciones y resolver situaciones de la profesión, al tener como base los problemas profesionales.

También las habilidades profesionales se consideran por Álvarez (2000), como:

El tipo de actividad que a lo largo del proceso de formación del profesional deberán sistematizarse hasta convertirse en una habilidad con un grado de generalidad tal, que le permita aplicar los conocimientos, actuar y transformar su objeto de trabajo y por lo tanto, resolver los problemas más generales y frecuentes que se presentan en las diferentes esferas de actuación (p.32).

Insiste, además, en la complejidad del trabajo del profesorado al tener que considerar quién enseña, qué enseña, cómo se enseña y el contexto social donde se desarrollan los procesos de enseñanza y aprendizaje. Se infiere, entonces, que las características distintivas de esta profesión deben sistematizarse en acciones y operaciones que los profesionales logren concientizar.

Álvarez (2000) señaló, sobre las habilidades profesionales, que constituyen la esencia de la actuación y a su juicio integran los conocimientos teóricos y prácticos y las habilidades menos complejas. Sus criterios resaltan la integración de los conocimientos para su formación y desarrollo en función de los problemas profesionales desde la formación inicial.

Las habilidades profesionales se analizan por Fuentes (2001) como:

El tipo de habilidad que a lo largo del proceso de formación del profesional deberá sistematizarse hasta convertirse en una habilidad con un grado de generalidad tal, que le permita aplicar los conocimientos, actuar y transformar su objeto de trabajo, y por lo tanto resolver los problemas más generales y frecuentes que se presenten en las esferas de actuación (p. 96).

Otros autores coinciden con los criterios anteriores: Lanuez y Pérez (2005) plantean que las habilidades profesionales permiten utilizar los conocimientos para solucionar tareas teóricas o prácticas, mientras que Rodríguez (2012) las considera como una dimensión del contenido y manifiestan el sistema de acciones intelectuales y prácticas las cuales facultan la solución de los problemas profesionales.

Por otra parte, Ruiz (2014) plantea que se forman en un proceso constructivo, socializado, en un espacio interdisciplinario, donde se integra lo académico, lo laboral y lo investigativo. Igualmente señala que sintetizan el ser, el saber y el hacer al desempeñarse en el objeto específico de la profesión y se enmarcan en la disposición de efectuar conscientemente las acciones en diferentes contextos.

A partir del análisis efectuado se asumen las posiciones de Álvarez (2000) al precisar las habilidades profesionales como un tipo de actividad que permite aplicar e integrar los conocimientos y otras habilidades menos complejas para actuar y transformar el objeto de trabajo.

$\mathrm{Al}$ referirse a la formación y el desarrollo de las habilidades profesionales Abdulina (1984) planteó que su dominio depende de la asimilación de los conocimientos teóricos y de las acciones prácticas en los cuales se basan. Destaca así la importancia de lograr un adecuado enfoque profesional pedagógico. También analizó como principios para su desarrollo los siguientes:

- El de la extensión y sistematicidad, que se concreta en el plan de estudio y se articula vertical y horizontalmente en todas las disciplinas.

- El de la combinación de la teoría y la práctica, que concibe la interrelación necesaria entre los conocimientos teóricos y la práctica.

- El del carácter jerárquico del Modelo del Profesional, que precisa los modos de actuación y permite derivar los objetivos a alcanzar.

Los autores asumen estos principios, pero consideran importante añadir la integración como uno de ellos, el cual se deberá expresar en las relaciones que se establecen entre las acciones y operaciones que conforman cada una de las invariantes y en su aplicación durante los diferentes temas de Didáctica de la Matemática. 
Se reconoce, entonces, que las habilidades profesionales forman parte del contenido a dominar por las y los estudiantes, sobre la base de los conocimientos de cada disciplina, y en consecuencia poder operar con estos al cumplir sus funciones como futuros educadores.

En este artículo se consideran las habilidades desde la teoría de la actividad, destacan el papel de las acciones y operaciones y sus relaciones entre sí, así como la importancia de formar modelos que acerquen a los sujetos al ideal aspirado durante su formación y desarrollo. Se reconoce que las habilidades profesionales son específicas de cada carrera y responden a una parte de la cultura que adquieren los sujetos en función de los problemas profesionales que deberán resolver.

\section{LA HABILIDAD PROFESIONAL PLANIFICAR LOS PROCESOS DE ENSEÑANZA Y APRENDIZAJE DE LA Matemática}

El posicionamiento teórico que fundamenta la habilidad profesional se sustenta en los criterios sobre la planificación desde las ciencias pedagógicas. En este sentido, la planificación se precisa por Remedios (2005) como:

El tratamiento metodológico que puede dársele a cada uno de los componentes, en función de identificar acciones que de modo procedimental permitan que desde este momento el docente modele didácticamente los contenidos que imparte, considerando las exigencias del currículo, las diferencias individuales y el contexto grupal, con una actuación independiente, crítica, reflexiva, para garantizar el protagonismo de sus estudiantes (p.17).

Esta autora describe el deber ser de la planificación como una etapa de los procesos de enseñanza y aprendizaje; aunque centra su análisis solo en el personal docente ya en ejercicio, sin tener en cuenta su etapa de formación inicial.

Resultan de interés las opiniones de Ginoris, Addine y Turcaz (2006) al ver la planificación como una labor permanente del docente donde se define un plan de acción flexible, que identifica los métodos y recursos, las tácticas y estructuras organizativas y metodológicas, así como el diseño de las estrategias para su ejecución; sin embargo, no revela el análisis de todos los componentes.

Para Ruiz (2007), la planificación consiste en determinar las influencias del profesorado en el estudiantado para el logro del aprendizaje y considera dos tipos: el fundamental, elaborado antes de la interacción alumnado-docente, y el regulador, confeccionado durante esta interacción. El presente escrito comparte esta idea, pero profundiza en el primero de los casos; pues una buena planificación asegura la ejecución de los procesos de enseñanza y aprendizaje y la calidad de sus resultados.

Especialistas en Didáctica de la Matemática como Lupiáñez y Rico (2008) precisan que la planificación establece las expectativas de aprendizaje e incorpora los criterios para su seguimiento y desarrollo, considerándose distintos niveles de dominio de las habilidades profesionales. Destaca así la influencia de la planificación en el posterior desarrollo del proceso y su efectividad en el aprendizaje. El propio Lupiáñez (2009) señala que la planificación es específica para cada tema de matemática y que se efectúa para un tiempo determinado, criterio el cual se enriquece al resaltar que es un proceso complejo donde se integran los contenidos desde la formación inicial.

Se plantea por Ruiz (2011) que planificar es:

Decidir por anticipado qué hacer, cuándo y dónde. Para esto hay que escoger entre todas las variantes existentes cuál es el medio para cumplir el objetivo. (...) se trata de definir a priori y partiendo de los objetivos, los contenidos necesarios, así como los métodos, medios y formas de enseñanza e incluso el tipo de evaluación. (p.15)

Aunque se asume este criterio por destacar el carácter rector del objetivo y sus relaciones con los demás componentes personalizados de los procesos de enseñanza y aprendizaje al desarrollar la planificación, es preciso señalar que no se explicita el papel del profesorado, estudiantes y del contexto sociocultural en el que se elaborará, aspectos de gran significación para los autores del artículo. 
$\mathrm{Al}$ analizar la planificación, especialistas en Didáctica de la Matemática como Jon, Fernández, Quintana y Carrasco (2002) la consideraron como un proceso complejo, no lineal que exige de la creatividad del y la docente; razón que hace necesario, desde la formación inicial, prestar especial atención a las habilidades profesionales correspondientes. Con vistas a perfeccionar la posición anterior, Álvarez, Almeida y Villegas (2014) plantearon que la dirección de los procesos de enseñanza y aprendizaje transita por cuatro fases las cuales se interrelacionan y complementan, según las funciones de la dirección: planificación, organización, ejecución y control.

Estos autores insisten en la planificación como proceso complejo, no esquemático y sin rigidez de pensamiento; e identifican como parte de la planificación el análisis metodológico de una unidad, el diseño de sistemas de clase y la planificación de clases; aunque estas no se analizan como habilidades profesionales a formar y desarrollar desde la Didáctica de la Matemática. A partir de esta realidad, se asume como principal resultado teórico de la investigación efectuada por Pérez (2015) la caracterización que elabora de la habilidad profesional objeto de análisis; al respecto plantea que planificar los procesos de enseñanza y aprendizaje de la Matemática:

Es una habilidad profesional que implica la integración de las invariantes: análisis metodológico de la unidad, diseño del sistema de clases y la planificación de clases; a partir de revelar las relaciones sistémicas entre los componentes personalizados y personales, aplicar las particularidades de las situaciones típicas, las líneas directrices, el enfoque metodológico de la asignatura y evidenciar actitudes propias de la profesión. (p.37)

Lo anterior implicó que el análisis metodológico de la unidad, el diseño del sistema de clases y la planificación de las clases se analizaran como invariantes de la habilidad profesional más integradora. En adelante se precisa el significado, así como las acciones y operaciones que deberá aprender a desarrollar el alumnado, futuro profesorado de Matemática; desde la posición del autor arriba citado.

Se considera de Pérez, Garriga y Valdés (2014) que la habilidad profesional para analizar metodológicamente la unidad consiste en:

El análisis personalizado de cada uno de los componentes del proceso de enseñanza-aprendizaje a partir de su carácter de sistema, de las particularidades del enfoque metodológico general de la asignatura, de las funciones didácticas, las situaciones típicas de la enseñanza de la Matemática, de las líneas directrices; logrando así una visión general de cómo cumplir de manera integrada con las aspiraciones de la unidad (p.9).

Este se ejecuta en colectivo, previo al diseño de los sistemas de clases. A partir del análisis de los elementos anteriores las acciones y operaciones que deben tomarse en cuenta al elaborar el análisis metodológico de las unidades de los programas escolares son las siguientes:

Este se ejecuta en colectivo, previo al diseño de los sistemas de clases. A partir del análisis de los elementos anteriores las acciones y operaciones que deben tomarse en cuenta al elaborar el análisis metodológico de las unidades de los programas escolares son las siguientes:

Acción 1: Análisis de los objetivos por cumplir y por diagnosticar en el estudiantado

Identificar los objetivos generales según los diferentes campos de la Didáctica de la Matemática; valorar el entrelazamiento de las líneas directrices y los elementos de la historia de la Matemática por resaltar; establecer relaciones entre los objetivos de la unidad, el grado, el nivel y de otras asignaturas e identificar variantes para diagnosticar los objetivos antecedentes, intereses y actitudes de las y los estudiantes.

Acción 2: Análisis del sistema de contenidos

Identificar los conceptos, teoremas y procedimientos matemáticos que se trabajan; identificar las habilidades matemáticas, las capacidades, hábitos, cualidades y convicciones que estimulan el tránsito a la independencia y la creatividad e identificar errores más frecuentes en el aprendizaje y sus posibles causas según las experiencias.

Acción 3: Selección de los métodos por utilizar 
Identificar los métodos que propicien la búsqueda de los contenidos y su comprensión, así como estilos de comunicación adecuados e identificar los procedimientos heurísticos y las estrategias cognitivas y metacognitivas que despierten la curiosidad científica y desafíen el razonamiento del estudiantado.

Acción 4: Selección de la bibliografía y de otros medios por utilizar

Identificar los libros de texto y los textos complementarios donde se desarrolle el contenido; identificar los medios de enseñanza-aprendizaje que pueden utilizarse, con énfasis en los recursos informáticos y precisar cómo pueden ser empleados para el desarrollo de los contenidos los resultados de investigaciones.

Acción 5: Elaboración de la dosificación de los contenidos

Determinar el tipo de clases y su tema, e identificar otras formas de organización que estimulen la ejecución de tareas interdisciplinarias diferenciadas.

Acción 6: Proyección de la estrategia de evaluación

Identificar los objetivos por evaluar según la evaluación frecuente, parcial o final y determinar las formas, vías de evaluación y medidas organizativas para evaluar los contenidos integradamente.

En la Figura 1 se muestran las relaciones conceptuales esenciales de la habilidad, así como sus acciones.

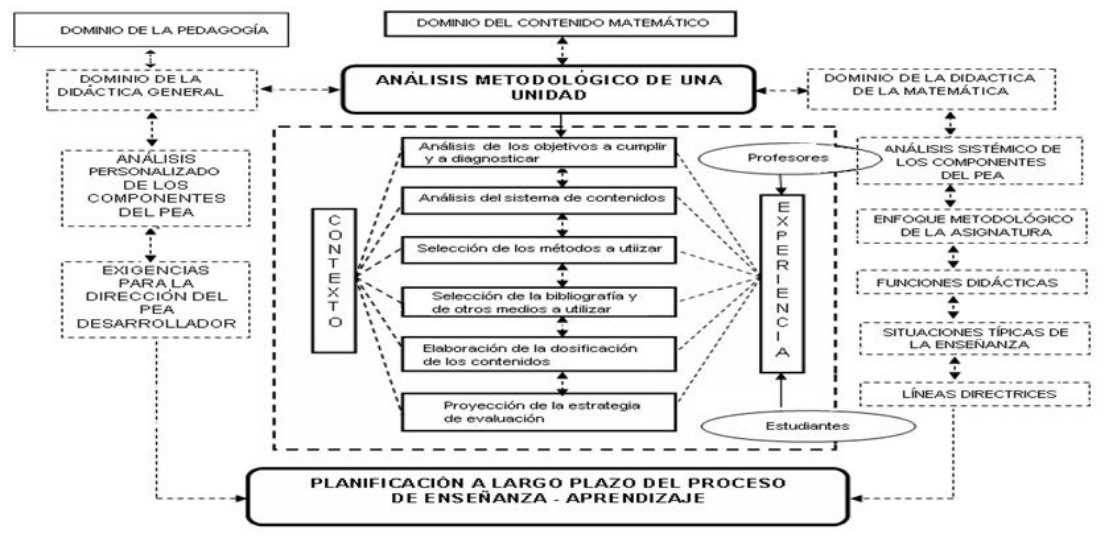

FIGURA 1

Representación gráfica de la habilidad profesional analizar metodológicamente unidades Fuente: Esquema elaborado para la investigación Pérez (2015).

De igual forma se consideran las posiciones de Pérez (2015) al referir que la habilidad profesional diseñar sistemas de clases implica:

Determinar el sistema de relaciones que se establecen entre los componentes personalizados y personales del proceso de enseñanza-aprendizaje de cada una de las clases vinculadas entre sí por sus potencialidades para el cumplimiento de uno o más objetivos parciales de la unidad y la lógica interna de su contenido y las particularidades del enfoque metodológico general de la asignatura, las funciones didácticas, las situaciones típicas, las líneas directrices. (p.41)

Esta se desarrolla en colectivo, previo a la planificación de las clases, en función de lograr objetivos parciales y parte del análisis metodológico de la unidad. Sus acciones y operaciones se relacionan a continuación:

Acción 1: Análisis de los objetivos del sistema de clases

Seleccionar los objetivos del sistema de clases según las situaciones típicas y la lógica interna del contenido; determinar los intereses y las actitudes a lograr; así como las condiciones externas del proceso e identificar los objetivos generales del sistema de clases y su contribución para la formación integral.

Acción 2: Determinación de los tipos de tareas por utilizar

Identificar tipos de tareas que impliquen: modelar, argumentar, transferir, comunicar y valorar situaciones representativas y elaborar otros tipos de tareas según los procesos parciales por los cuales transita la situación típica que predomine.

Acción 3: Diseño a grosso modo de cada clase 
Determinar el objetivo específico según la habilidad, los conocimientos y sus potencialidades educativas; seleccionar los tipos de tareas y las situaciones por utilizar para la organización interna de la clase; precisar posibles métodos, procedimientos y formas de organización según los tipos de tareas; determinar o prever la elaboración de los medios de enseñanza-aprendizaje según los tipos de tareas por emplear y determinar las características de los instrumentos por usar para evaluar los objetivos de cada clase y del sistema, con miras en los objetivos de la unidad.

En la Figura 2 se presentan las relaciones conceptuales esenciales de la habilidad, así como sus acciones.

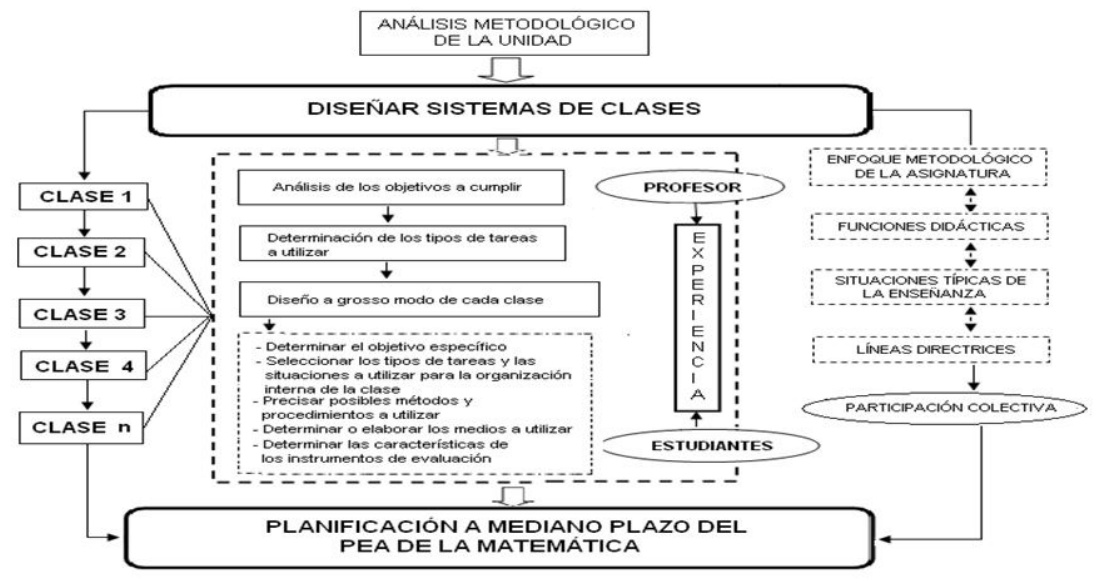

FIGURA 2

Representación gráfica de la habilidad profesional diseñar sistemas de clases

Fuente: Esquema elaborado para la investigación Pérez (2015)

Por último, se comparten los criterios de Pérez (2015) en relación con la habilidad profesional planificar clases, la cual a su juicio implica:

Prever, de forma más cercana al acto de la clase, las relaciones que se establecen entre los componentes del proceso de enseñanza-aprendizaje, según las ideas reflejadas en el sistema de clases y el análisis metodológico de la unidad. En ellas se expresan las tareas docentes, con su correspondiente estructuración didáctica y otras medidas organizativas que permiten al profesor y los estudiantes y, a estos entre sí, interactuar en función de la búsqueda de los nuevos conocimientos y su fijación, cumpliendo con el enfoque metodológico general de la asignatura, las funciones didácticas, la situación típica y la línea directriz que predomine. (p. 42)

Se desarrolla de forma individual, previa a la ejecución en el aula con la intención de asegurar su calidad. Se describen las acciones y operaciones por ejecutar, expresando las relaciones que entre ellas se manifiestan.

Acción 1: Determinar las actividades por desarrollar durante la introducción

Elaborar las preguntas por utilizar durante la revisión del estudio independiente, para asegurar desde el nivel de partida, la comprensión del contenido matemático y su significado práctico y determinar las problemáticas matemáticas o extra matemáticas a partir de las cuales se orienta el objetivo y se motiva la clase, destacando el contenido desconocido y su importancia para resolver nuevas clases de problemas.

Acción 2: Determinar las actividades por ejecutar durante el desarrollo

Si la clase es de tratamiento de la nueva materia las operaciones por ejecutar son: seleccionar o elaborar las tareas docentes por utilizar para la introducir el contenido según la situación típica, los métodos, medios, formas de organización y evaluación por emplear; seleccionar o elaborar ejemplos, ejercicios y/o problemas para propiciar la asimilación inicial del contenido e ilustrar su aplicación práctica y elaborar las preguntas e impulsos que propicien la búsqueda y elaboración del nuevo contenido, el autoconocimiento y regulación de la actuación. 
Si la clase es de fijación, entonces las operaciones por organizar son: Seleccionar, diseñar y resolver los ejercicios y/o problemas para fijar el conocimiento y desarrollar las habilidades, según la situación típica y los métodos, medios, formas de organización y evaluación por utilizar y elaborar las preguntas complementarias e impulsos que puedan enriquecer el análisis de los ejercicios y/o problemas propuestos para el trabajo independiente y su valoración por el estudiantado.

Acción 3: Determinar las actividades por desarrollar durante las conclusiones

Organizar el sistema de preguntas para comprobar el cumplimiento de los objetivos de manera que se analice lo esencial del contenido y sus posibilidades de transferencia; seleccionar, elaborar y resolver los ejercicios y/o problemas que orientará de estudio independiente y prever la valoración de la actuación grupal y de algunos y algunas estudiantes seleccionados/as, así como las posibles acciones correctivas y estrategias de aprendizaje a desarrollar.

La Figura 3 detalla las relaciones conceptuales esenciales de la habilidad, así como sus acciones.

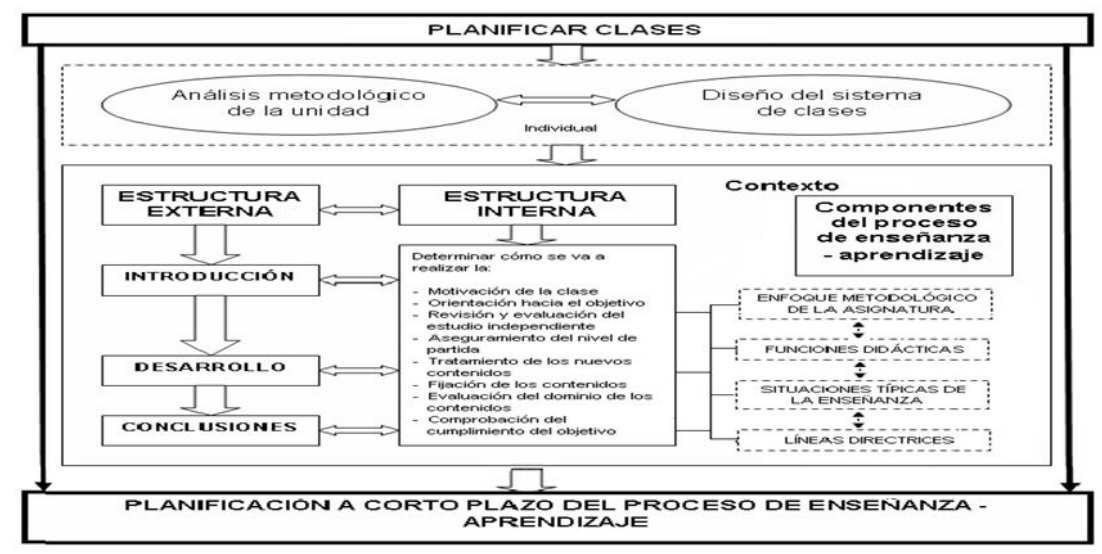

FIGURA 3

Representación gráfica de la habilidad profesional planificar clases

Fuente: Esquema elaborado para la investigación Pérez (2015)

\section{ESTRATEGIA DIDÁCTICA PARA LA FORMACIÓN Y DESARROLLO DE LA HABILIDAD PROFESIONAL PLANIFICAR LOS PROCESOS DE ENSEÑANZA Y APRENDIZAJE DE LA MATEMÁtiCA. RESULTADOS DE SU APLICACIÓN PRÁCTICA}

Esta estrategia didáctica se diseñó para la formación inicial del estudiantado de la carrera Licenciatura en Educación, especialidad Matemática-Física. La misma se genera desde la disciplina Didáctica de la Matemática.

Según la intención del artículo, los autores asumen la definición de estrategia didáctica dada por Rodríguez y Rodríguez (2011) donde se analiza como:

La proyección de un sistema de acciones a corto, mediano y largo plazo que permite la transformación del proceso de enseñanza-aprendizaje en una asignatura, nivel o institución tomando como base los componentes del mismo y que permite el logro de los objetivos propuestos en un tiempo concreto. (p. 39)

Además, se comparte el criterio de Zúñiga (2017) al reconocer que toda estrategia didáctica constituye un procedimiento organizado, formalizado y orientado para la obtención de una meta y que al aplicarla en la práctica se requiere de su perfeccionamiento por parte de los docentes.

Seguidamente se describe la estrategia comenzando por sus características, exigencias, objetivo general, etapas y las acciones fundamentales de cada una de ellas; así como los resultados de su aplicación. La estrategia 
se caracteriza por ser: objetiva, flexible, participativa e integradora; esta última vista como su cualidad distintiva.

La objetividad está dada por su diseño consciente en función de la solución de un problema profesional constatado en la formación inicial del profesorado de Matemática. Para ello se estudiaron los documentos rectores de la carrera y los resultados del diagnóstico del estudiantado.

La flexibilidad se expresa en la posibilidad de rediseñar sus acciones según los resultados que se obtienen en tanto se forma y se desarrolla la habilidad profesional; de igual modo las orientaciones que se ofrecen para implementarlas se pueden perfeccionar según las necesidades del alumnado y las relaciones que se logren establecer entre las disciplinas del plan de estudio y desde todos los componentes.

Su carácter participativo parte de reconocer la responsabilidad de todos sus participantes en la formación y el desarrollo de la habilidad profesional y en que estimula el intercambio sistemático y cooperado de ellos al ejecutar las acciones.

El carácter integrador se manifiesta al jerarquizar, durante el tratamiento de los contenidos de Didáctica de la Matemática, las relaciones entre las invariantes de la habilidad profesional que se pretende formar y desarrollar. Esto permite, a la vez que se estudian las situaciones típicas y las líneas directrices para la enseñanza de la Matemática, continuar aprendiendo sobre la planificación.

Las exigencias que guían la estrategia didáctica son las siguientes: profundizar en el contenido de los programas escolares como recurso imprescindible para la planificación; vincular la teoría y la práctica, mediante la integración de las invariantes de la habilidad profesional; favorecer la implicación, satisfacción y disposición de las y los estudiantes, desde el trabajo individual y colectivo, por las actividades de planificación y garantizar la elaboración y utilización de tareas docentes que faciliten la integración de las invariantes de la habilidad profesional que se analiza.

Según estas posiciones el objetivo general de la estrategia didáctica es: perfeccionar la formación y el desarrollo de la habilidad profesional planificar los procesos de enseñanza y aprendizaje de la Matemática, a partir de la integración de sus invariantes desde la disciplina Didáctica de la Matemática en la carrera Licenciatura en Educación, Matemática-Física. Para su cumplimiento se consideran cuatro etapas interrelacionadas entre sí; que se diferencia solo para su presentación. Ellas son: diagnóstico, planificación, instrumentación y evaluación.

El objetivo del diagnóstico fue: determinar el dominio de los contenidos necesarios para la planificación de los procesos de enseñanza y aprendizaje.

Acciones por desarrollar: diagnóstico del dominio de los contenidos de la matemática escolar y de la didáctica, diagnóstico del dominio del enfoque metodológico general de la Matemática, discusión de los resultados en los colectivos pedagógicos y estudiantil y proyección de acciones correctivas en función de las debilidades identificadas.

Se orienta a los docentes, que al efectuar el diagnóstico deben:

- Profundizar en el dominio de los conocimientos matemáticos y didácticos; sus dificultades, errores más frecuentes y posibles causas.

- Valorar si logran explicar las relaciones entre los componentes y la estructura interna de la clase, si diseñan tareas docentes que cumplan con el enfoque metodológico general y si reconocen sus ideas metodológicas esenciales.

- Prestar atención a la disposición y satisfacción por aprender a planificar los procesos de enseñanza y aprendizaje e implicar al estudiantado en el análisis de sus fortalezas y dificultades para lograr la solución de estas últimas.

- Identificar y analizar, en el colectivo de año, las fortalezas y debilidades que influyen en la formación y el desarrollo de la habilidad profesional que se analiza para el diseño de las acciones de atención individualizada. 
Una vez puestas en práctica estas acciones y aplicadas las técnicas correspondientes (revisión del producto de la actividad y la observación pedagógica) fue posible identificar una tendencia positiva en relación con el dominio que posee el alumnado de los contenidos de la matemática escolar y de la didáctica (con una media aritmética superior a los 4,18 puntos); así como del enfoque metodológico general de esta asignatura (con una media aritmética superior a los 4,09 puntos).

Del análisis de los resultados, por parte de los docentes, se derivaron acciones correctivas en función de asegurar una mayor integración de los contenidos correspondientes; así como una argumentación más efectiva y reflexiva de las soluciones dadas a las tareas docentes resueltas cada estudiante.

Igualmente, el hacer partícipe consciente al estudiantado del análisis de sus fortalezas y debilidades, les permitió a sí mismos una implicación mayor en el proceso de formación inicial y, en particular, favoreció su implicación en las acciones relacionadas con el aprender a planificar los procesos de enseñanza y aprendizaje.

Posteriormente, el objetivo por cumplir durante la planificación fue: diseñar las acciones para la formación y el desarrollo de la habilidad profesional correspondiente.

Acciones por ejecutar: análisis de las relaciones entre los componentes de los procesos de enseñanza y aprendizaje según las exigencias, determinación de los tipos de tareas docentes a utilizar para enseñar a planificarlo, elaboración de las tareas docentes que integren las invariantes de la habilidad profesional a formar y desarrollar y diseño de la preparación de las y los docentes en temas relacionados con la planificación.

Al planificar la formación y desarrollo de la habilidad profesional los docentes deben:

- Determinar, para cada año, los subproblemas profesionales a resolver y, a partir de ellos, precisar los objetivos específicos a cumplir explicitando la necesidad de integrar las invariantes de la habilidad profesional.

- Considerar la conceptualización de la habilidad profesional, sus invariantes y las acciones y operaciones por efectuar como uno de los conocimientos.

- Estimular una mayor implicación, satisfacción y disposición por las actividades de planificación a partir de utilizar métodos que inciten el uso de los procedimientos heurísticos y las formas de trabajo y de pensamiento.

- Utilizar los medios que existen para aprender a planificar y los que organizan y orientan la enseñanza.

- Potenciar un clima afectivo el cual estimule la implicación y satisfacción de las y los estudiantes en todas las formas de organización; en particular, la práctica laboral.

- Diferenciar tipos de tareas docentes las cuales en la práctica se complementen: académicas, laborales e investigativas.

- Prever las acciones de preparación para que el conjunto docente dirija la formación y el desarrollo de la habilidad profesional.

El principal resultado de las acciones anteriores se expresó en el diseño de las tareas docentes. A continuación, se muestran ejemplos de las utilizadas.

1. Consulte uno de los programas escolares de Matemática e identifique en sus orientaciones metodológicas ideas que:

a) Ejemplifiquen las potencialidades educativas de los contenidos matemáticos.

b) Demuestren la relación existente entre los objetos matemáticos (conceptos, teoremas y procedimientos) que se estudian y la sociedad. Argumente.

c) Destaquen las potencialidades de los contenidos para cumplir con el enfoque metodológico general de la asignatura.

2. Analice y resuelve los siguientes ejercicios y problemas. 
- Determine para qué valores de $\mathrm{k}$ tiene la ecuación: $\mathrm{x}^{2}+6 \mathrm{x}+\mathrm{k}=0 \mathrm{y} \mathrm{x}^{2}+\mathrm{kx}+9=\mathrm{dos}$ soluciones, una solución o ninguna solución real. (Ejercicio 7 pág. 15 Libro de Texto Complementario Matemática 10. Grado).

- Una juguetería tiene una ganancia del $40 \%$ por cada juguete que vende. Encuentre el precio de venta de una pelota de balompié que costó $\$ 11,00$. Si el vendedor obtuvo una ganancia de $\$ 10,00$ cuando vendió una pistola. Calcule el precio de venta de este juguete.

- En la figura el triángulo $\mathrm{ABC}$ es rectángulo en $\mathrm{A}, \mathrm{DE} \# \mathrm{AB}$. Clasifique el triángulo $\mathrm{ABC}$ según la longitud de sus lados.

a) Explique la o las vías de solución utilizada en cada caso y los diferentes tipos de contenidos por utilizar.

b) Explique las potencialidades de cada uno de ellos para la formación integral de la personalidad de los sujetos.

c) ¿A qué lineamiento del enfoque metodológico de la asignatura se le puede dar cumplimiento con cada uno de los ejercicios y problemas anteriores? Explique.

3. A partir de los sistemas de clases seleccionados, estudie el programa de la asignatura, el libro de texto del grado y las ideas reflejadas en el análisis metodológico de la unidad y efectúe las siguientes actividades:

a) Identifique todos los tipos de tareas por utilizar en cada uno.

b) Determine cuáles de los procesos matemáticos se deberán desarrollar en cada sistema de clases.

c) Seleccione los ejercicios y problemas que respondan a los diferentes niveles de asimilación y a los tipos de tareas por utilizar en cada sistema de clases.

d) ¿Cuáles son los errores más frecuentes en el aprendizaje de los contenidos de cada sistema de clase? ¿Qué hacer para evitarlos?

4. Teniendo en cuenta las clases seleccionadas elabore en cada caso:

a) La tarea docente para la motivación. Precise si es intra o extra matemática.

b) La tarea docente o el sistema de preguntas para asegurar el nivel de partida. Tenga en cuenta la comprensión del contenido y su significado práctico.

c) El sistema de preguntas complementarias para la revisión del estudio independiente. Trate de establecer, si es posible, relaciones entre los contenidos que permitan estimular la independencia cognoscitiva.

d) La problemática o interrogantes que puede utilizar para orientar el objetivo luego de identificar el contenido desconocido y su importancia.

Además, se trabajó en la preparación del cuerpo docente participante en temas relacionados con la planificación. En particular, se impartieron los siguientes temas: El enfoque metodológico de la asignatura Matemática. Tendencias actuales de la Didáctica de la Matemática y La planificación de los procesos de enseñanza y aprendizaje y su carácter integrador.

El profesorado manifestó una tendencia favorable hacia los temas antes referidos y valoraron de positivos los materiales didácticos elaborados para utilizar durante la formación y el desarrollo de la habilidad planificar los procesos de enseñanza y aprendizaje. Se mostraron satisfechos con la pertinencia de las acciones de la estrategia y reconocieron la posibilidad de su aplicación y mejora sistemática.

La tercera etapa, dedicada a la instrumentación de la estrategia, tuvo como objetivo: implementar las acciones para la formación y el desarrollo de la habilidad profesional correspondiente. 
Acciones por ejecutar: preparación del profesorado en los temas seleccionados y otros que resulten necesarios; ejecución de las clases, según el momento de formación y desarrollo de la habilidad profesional y el diagnóstico del alumnado; desarrollo de sesiones de práctica que complementen la formación y el desarrollo de la habilidad profesional y elaboración de consultas y prácticas de estudio para la atención diferenciada según las dificultades.

Como parte de la ejecución de las acciones previstas se debe:

- Preparar al conjunto docente participante en el enfoque metodológico general de la asignatura y en la conceptualización de la habilidad profesional que se trabaja.

- Utilizar la caracterización de la habilidad propuesta y enfatizar en las relaciones entre cada una de sus invariantes y aplicarlas al tratamiento de las situaciones típicas y las líneas directrices.

- Introducir temas afines a la planificación como: la diferenciación, la evaluación y el trabajo metodológico y promover, en los seminarios, la elaboración de resúmenes, de mapas conceptuales y de fichas de contenido.

- Organizar talleres que permitan aplicar los contenidos a partir de la orientación previa de trabajos extra clase y promuevan la participación activa de las y los estudiantes en los espacios de dirigidos a la preparación de la asignatura.

- Efectuar consultas, sesiones de tutoría y prácticas de estudio en las cuales se fortalezca la preparación del estudiantado para la planificación.

Este momento ocurrió durante la ejecución de las actividades docentes de la disciplina Didáctica de la Matemática y desde las sesiones de la práctica laboral que desarrollaba el alumnado.

Para el grupo docente constituyó un espacio de retroalimentación el cual facilitó el perfeccionamiento de las tareas docentes elaboradas y de las acciones de la estrategia en sí. Igualmente, el seguimiento de las y los estudiantes mostró avancen positivos en relación al proceso de formación y desarrollo de la habilidad correspondiente, resultados que se explican más adelante.

Por último, el objetivo de la evaluación siempre fue: valorar los resultados de la aplicación de la estrategia didáctica para conocer su efectividad en la práctica.

Acciones por efectuar: análisis del nivel de formación y desarrollo de la habilidad profesional que se analiza en las y los estudiantes, perfeccionamiento de las acciones elaboradas y propuesta de nuevas acciones en función de los resultados.

Como parte de la evaluación de la efectividad de la estrategia los docentes deben:

- Aplicar a través de la autoevaluación el cumplimiento del o los objetivos específicos de cada actividad.

- Enfatizar en la efectividad de las tareas docentes para la contextualización y sistematización de los contenidos y el intercambio de experiencias y prestar atención al dominio de los contenidos matemáticos y los didácticos.

- Atender a la implicación, satisfacción y disposición que muestra el estudiantado al efectuar las actividades.

Para la evaluación de la estrategia se aplicaron los métodos descritos al precisar la metodología y se tuvieron en cuenta las siguientes dimensiones: dominio de las acciones y operaciones por desarrollar durante la planificación (D1), aplicación de las acciones y operaciones por ejecutar durante la planificación según las particularidades del contenido matemático (D2), relación establecida entre las habilidades para la planificación (D3) y agrado mostrado por las y los estudiantes durante las actividades relacionadas con la planificación (D4).

Se elaboró el análisis del producto de la actividad a veintidós trabajos de las y los nueve estudiantes de la muestra. De ellos, cinco análisis metodológicos, siete sistemas de clases y diez clases. También se observaron 
doce actividades docentes (clases y sesiones de práctica laboral) en las que se analizó el trabajo con las habilidades correspondientes. La Tabla 1 muestra el resumen de los datos obtenidos.

TABLA 1

Resultados de los instrumentos aplicados para valorar la formación y desarrollo de la habilidad profesional planificar los procesos de enseñanza y aprendizaje.

\begin{tabular}{|c|c|c|c|c|c|}
\hline \multirow{3}{*}{$\begin{array}{l}\text { Dimensiones } \\
\text { evaluadas }\end{array}$} & \multicolumn{4}{|c|}{ Frecuencia Absoluta por Categorías } & \multirow{3}{*}{$\begin{array}{l}\text { Media } \\
\text { Aritmética }\end{array}$} \\
\hline & E (5) & MB (4) & B (3) & M (2) & \\
\hline & \multicolumn{4}{|c|}{ Análisis del producto de la actividad } & \\
\hline D1 & 7 & 12 & 3 & & 4.18 \\
\hline $\mathrm{D} 2$ & 6 & 12 & 2 & 2 & 4.00 \\
\hline \multirow[t]{2}{*}{ D3 } & 5 & 14 & 2 & 1 & 4.05 \\
\hline & \multicolumn{5}{|c|}{ Observación pedagógica } \\
\hline D1 & 4 & 5 & 3 & & 4.08 \\
\hline $\mathrm{D} 2$ & 5 & 5 & 1 & 1 & 4.17 \\
\hline D3 & 3 & 7 & 1 & 1 & 4.00 \\
\hline D4 & 9 & 2 & 1 & & 4.67 \\
\hline
\end{tabular}

Fuente: Elaboración propia

El análisis de la media aritmética de cada dimensión en ambos instrumentos muestra un valor superior a los cuatro puntos, lo cual indica que predominan las evaluaciones de excelente y muy bien, según la escala utilizada. Lo anterior evidencia la validez de la estrategia didáctica que se propone.

Las y los estudiantes además confirmaron que la caracterización de la habilidad planificar los procesos de enseñanza y aprendizaje utilizada como soporte principal de la estrategia didáctica constituyó un elemento importante en su formación profesional.

\section{CONCLUSIONES}

El estudio teórico permitió determinar que las habilidades surgen de la actividad, mediante un proceso de formación y desarrollo que exige de la precisión de las acciones y operaciones que las conforman y de su integración en variados contextos.

Las habilidades profesionales resultan distintivas de cada profesión, su formación y desarrollo exigen de la integración de los conocimientos teóricos y prácticos.

La habilidad profesional planificar los procesos de enseñanza y aprendizaje es analizada como una habilidad profesional compleja que precisa de tres habilidades profesiones que se integran entre sí; ellas son: el análisis metodológico de la unidad, el diseño de sistemas de clases y la planificación de clases.

La estrategia didáctica diseñada para el contexto de la formación inicial del profesorado de Matemática logra organizar el proceso de formación y desarrollo de la habilidad profesional planificar el proceso de enseñanza y aprendizaje, a partir de tener en consideración las particularidades de la didáctica de esta asignatura.

Los resultados de la aplicación de la estrategia didáctica se reflejaron en el nivel de formación y desarrollo de dicha habilidad en las y los estudiantes de la muestra. Así como en los planteamientos de los docentes participantes en relación a la factibilidad de su empleo en diversos contextos.

\section{REFERENCIAS}

Abdulina, O. A. (1984). La preparación pedagógica general del maestro en el sistema de instrucción pedagógica. Moscú: Prosvechenie. 
Álvarez, C. M. (1988). Fundamentos teóricos de la dirección del proceso de formación del profesional de perfil amplio. Villa Clara, Cuba: Universidad Central de Las Villas.

Álvarez, C. M. (2001). Diseño Curricular. La Habana, Cuba: Pueblo y Educación.

Álvarez, R. M. (2000). La didáctica de las Ciencias Sociales: Eje de la formación del profesorado. En Pagés I., Blanch, J., Estepa Jiménez, J. y Través González, G., Modelos, contenidos y experiencias en la formación de profesores de Ciencias Sociales (pp. 151-156). España: Universidad de Huelva.

Álvarez, M., Almeida, B. y Villegas, E. V. (2014). El proceso de enseñanza-aprendizaje de la asignatura matemática documentos metodológicos. La Habana: Editorial Pueblo y Educación.

Barreras, F. (1997). Programa del módulo "Modelo pedagógico para la formación y desarrollo de habilidades, hábitos y capacidades”. La Habana, Cuba: Instituto Pedagógico Latinoamericano y Caribeño.

Barreras, F. (2003). Material docente básico del curso modelo pedagógico para la formación y desarrollo de habilidades, hábitos y capacidades. La Habana, Cuba: Instituto Pedagógico Latinoamericano y Caribeño.

Bermúdez, R. y Rodríguez, M. (1996). Teoría y metodología para el aprendizaje. La Habana, Cuba: Pueblo y Educación. Brito, H. (1984). Hábitos, habilidades y capacidades. Revista Varona, 13, 73-88.

Danilov, M. (1985). El proceso de enseñanza en la escuela. La Habana: Libros para educación.

Fuentes, H. C. (2001). Didáctica de la Educación Superior. Santiago de Cuba, Cuba: Universidad de Oriente

Fuentes, H. C. y Álvarez, I. B. (1998). Dinámica del proceso docente educativo de la Educación Superior. Santiago de Cuba, Cuba: Centro de Estudios de Educación Superior. Universidad de Oriente

Ginoris, O., Addine, F. y Turcaz, J. (2006). Curso Didáctica General. La Habana, Cuba: Instituto Pedagógico Latinoamericano y Caribeño.

González, M. C., Vega, F. y Francisco, L. (2015). Las habilidades profesionales pedagógicas en la formación de profesores de Matemática-Física. Curso 27. Pedagogía 2015. La Habana, Cuba: Educación Cubana.

González, V., Castellanos, D., Córdova, M. D., Rebollar, M., Martínez, M., Fernández, A. M., Pérez, D. (1995). Psicología para Educadores. La Habana, Cuba: Pueblo y Educación.

Jon, M., Fernández, J., Quintana, A. y Carrasco, A. (2002). El Tratamiento Metodológico de una Unidad y de un Sistema de Clases. En El transcurso de las Lineas Directrices y la Planificación de la enseñanza en los Programas de Matemática de los municipios seleccionados para las Transformaciones del Programa de las Secundarias Básicas. La Habana: Editorial Pueblo y Educación.

Lanuez, M. del C. y Pérez, V. (2005). Habilidades para el trabajo investigativo: experiencias en el Instituto Pedagógico Latinoamericano y Caribeño (IPLAC). CD Pedagogía 2005. La Habana, Cuba: Educación Cubana.

Lerner, I. Y. y Skatkin, M. N. (1980). Tareas y contenido de la enseñanza general y politécnica. En M. A. Danilov y otros (Eds). Didáctica de la escuela media. La Habana, Cuba: Libros para la educación.

López, M. (1990). Sabes enseñar a describir, definir y argumentar. La Habana, Cuba: Pueblo y Educación.

Lupiáñez, J. L. y Rico, L. (2008). Análisis didáctico y formación inicial de profesores: competencias y capacidades en el aprendizaje de los escolares. PNA, 3(1), 35-48.

Lupiáñez, J. L. (2009). Expectativas de aprendizaje y planificación curricular en un programa de formación inicial de profesores de matemáticas de secundaria (Tesis de doctorado). Universidad de Granada, Granada.

Márquez, A. (1987). Las Habilidades Pedagógicas. Santiago de Cuba, Cuba: Material inédito

Massón, R. M., Llivina, M. J. y Arencibia, V. (2011). Estudio comparado en la formación de profesores de Secundaria Básica. La Habana, Cuba: Educación Cubana.

Miari, A. (1982). Organización y metodología de la enseñanza práctica. La Habana, Cuba: Pueblo y Educación.

Pérez, A., Garriga, T. y Valdés, M. (2014). Procedimiento para la realización del tratamiento metodológico de la unidad. Exigencias desde la formación inicial del profesor de matemática. Revista IPLAC, 18(1), $42-55$.

Pérez, A. (2015). La integración de las invariantes de la habilidad profesional planificar el proceso de enseñanzaaprendizaje desde la Didáctica de la Matemática (tesis de doctorado). Universidad "José Martí Pérez", Sancti Spíritus, Cuba. 
Petrovski, A. V. (1980). Psicología evolutiva y pedagógica. Moscú: Progreso.

Remedios, J. M. (2005). Desempeño profesional y evaluación de los docentes del Instituto Superior Pedagógico: propósitos y perspectivas. Sancti Spíritus, Cuba: Universidad de Ciencias Pedagógicas "Silverio Blanco Núñez".

Rodríguez, M. A. y Rodríguez, A. (2011). La estrategia como resultado científico de la investigación educativa. En: N. De Armas Ramírez y A. Valle Lima, Resultados cientificos en la investigación educativa (pp. 20-51). La Habana, Cuba: Pueblo y Educación.

Rodríguez, I. (2012). Conceptualización y estructura de las habilidades profesionales básicas del técnico de nivel medio en agronomia. Recuperado de https://bit.ly/2Yz3jB2

Ruiz, A. M. (2007). La integración de conceptos matemáticos a partir de las relaciones conceptuales clásicas en la educación preuniversitaria (tesis de doctorado). Universidad de Ciencias Pedagógicas "Silverio Blanco Núñez", Sancti Spíritus, Cuba.

Ruiz, J. M. (2011). Fundamentos teóricos y metodológicos de la dirección y la gestión cientifica de los procesos universitarios. En J. M. Ruiz (Ed.) La gestión universitaria y el rol del profesor. (pp. 11-12). La Habana: Félix Varela.

Ruiz, O. (2014). La formación de las habilidades profesionales pedagógicas en los estudiantes de la Licenciatura en Educación Especialidad Pedagogía-Psicología (Tesis de doctorado). Universidad de Ciencias Pedagógicas "Félix Varela Morales", Villa Clara, Cuba.

Saravia, L. M. y Flores, I. (2005). La formación de maestros en América Latina. Estudio realizado en diez países. Recuperado de http://repositorio.minedu.gob.pe/handle/123456789/480

Savin, N. V. (1972). Pedagogía. La Habana, Cuba: Pueblo y Educación.

Talízina, N. F. (1988). Psicología de la enseñanza. Moscú: Progreso.

Zúñiga, M. (2017). La estrategia didáctica: Una combinación de técnicas didácticas para desarrollar un plan de gestión de riesgos en la clase. Revista Educación 41(1), 1-18

\section{BY-NC-ND}

